\title{
Constraining Forces Causing the Meissner Effect
}

\section{Ekkehard Krüger}

Institut für Materialwissenschaft, Materialphysik, Universität Stuttgart, Stuttgart, Germany

Email: ekkehard.krueger@imw.uni-stuttgart.de

How to cite this paper: Krüger, E. (2017) Constraining Forces Causing the Meissner Effect. Journal of Modern Physics, 8, 11341142.

https://doi.org/10.4236/jmp.2017.88074

Received: April 28, 2017

Accepted: June 25, 2017

Published: June 28, 2017

Copyright $\odot 2017$ by author and Scientific Research Publishing Inc.

This work is licensed under the Creative

Commons Attribution International

License (CC BY 4.0).

http://creativecommons.org/licenses/by/4.0/

\begin{abstract}
As shown in former papers, the nonadiabatic Heisenberg model presents a novel mechanism of Cooper pair formation which is not the result of an attractive electron-electron interaction but can be described in terms of quantum mechanical constraining forces. This mechanism operates in narrow, roughly half-filled superconducting bands of special symmetry and is evidently responsible for the formation of Cooper pairs in all superconductors. Here we consider this new mechanism within an outer magnetic field. We show that in the magnetic field the constraining forces produce Cooper pairs of non-vanishing total momentum with the consequence that an electric current flows within the superconductor. This current satisfies the London equations and, consequently, leads to the Meissner effect. This theoretical result is confirmed by the experimental observation that all superconductors, whether conventional or unconventional, exhibit the Meissner effect.
\end{abstract}

\section{Keywords}

Superconductivity, Meissner Effect, Nonadiabatic Heisenberg Model, Time Inversion in a Magnetic Field, Constraining Forces

\section{Introduction}

The nonadiabatic Heisenberg model [1] (NHM) emphasizes the picture of strongly correlated atomic-like electrons in nearly half-filled narrow energy bands. Within the NHM, the appertaining localized states are consequently represented by symmetry-adapted and optimally localized Wannier functions. In some metals, these Wannier functions must be chosen spin-dependent in order that they are both symmetry-adapted and optimally localized [2]. An energy band with such spin-dependent Wannier functions is called "superconducting band" because only metals possessing a narrow, roughly half-filled superconducting band experimentally prove to be (conventional, high- $T_{c}$ or other) 
superconductors, see the Introduction of Ref. [2]. This observation can be interpreted straightforwardly within the NHM [3]. Within this model, the formation of Cooper pairs is not the result of an attractive electron-electron interaction but may be described in terms of quantum mechanical constraining forces operating in superconducting bands. There is evidence that these constraining forces are necessary for the Hamiltonian of the system to possess superconducting eigenstates, see, e.g., Section 6 of Ref. [4]. This applies to all superconductors, whether conventional or unconventional.

Also within the NHM, the formation of Cooper pairs is mediated by bosons, which, however, bear the crystal spin angular momentum $1 \cdot \hbar$. More precisely, the electrons couple to the energetically lowest boson excitations of the crystal that possess the crystal-spin angular momentum $1 \cdot \hbar$ and are sufficiently stable to transport it through the crystal [5]. This distinguishes the theory of superconductivity within the NHM from the standard theory. The superconducting transition temperature $T_{c}$ is determined by the excitation energy of the crystal-spin-1 bosons mediating the pair formation. As is well-known, the kinetic energy of particles is not changed by constraining forces (and, hence, they can easily be overlooked). Thus, also in a superconducting band, $T_{c}$ is determined by the standard theory of superconductivity. In particular, in the isotropic elemental superconductors (often referred to as "conventional" superconductors) pure phonons are able to carry crystal-spin-1 angular momentum [5] [6]. Thus, the transition temperature of the elemental superconductors is still defined by the Bardeen-Cooper-Schrieffer theory [7].

The aim of this paper is to provide evidence that the constraining forces causing the formation of Cooper pairs in superconducting bands are also responsible for the Meissner effect. When superconductors are cooled below their transition temperature $T_{c}$, they not only lose their electrical resistance but also create currents which completely oppose an applied magnetic field. This second effect was discovered 1933 by Meissner and Ochsenfeld [8] and is generally referred to as Meissner-Ochsenfeld effect or, shortly, Meissner effect. J.E. Hirsch [9] argued that a mechanism proposed to explain superconductivity must also explain the Meissner effect because this effect is observed in all superconductors. We show that the mechanism of Cooper pair formation defined within the NHM meets this strict requirement of Hirsch.

However, we do not explain the Meissner effect but we restrict ourselves to derive the London equations [10] which are generally believed to explain the Meissner effect [11] (though they are partially called into question by Hirsch, see Section 6). In the following Section 2, we briefly explain the mechanism of Cooper pair formation within the NHM. In particular, we outline the important role of both constraining forces and the time-inversion symmetry in the formation of Cooper pairs. In Section 4.1, we define the "inner time-inversion" within an external magnetic field, in Section 4.2 we will derive Equation (19) giving the total momentum of a Cooper pair in an outer magnetic field, and in Section 5 we shall derive the London equations. 


\section{Cooper-Pair Formation in a Superconducting Band}

The mechanism of the Cooper pair formation in a narrow, roughly half-filled superconducting band has been described in a former paper [3]. In this section we give a short overview of the features of this mechanism necessary for an understanding of the Meissner effect. For a more detailed summary see Section 3 of Ref. [4].

\subsection{Superconducting Band in the Absence of a Magnetic Field}

First we assume no outer magnetic field to be present. The Bloch functions of a superconducting band can be unitarily transformed into optimally localized spin-dependent Wannier functions which are adapted to the symmetry of the electron system [2]. In this context, the "symmetry of the electron system" also comprises the time-inversion symmetry. The NHM defines atomic-like electrons with localized states represented by these spin-dependent Wannier functions. As a consequence of their spin dependence, the spin directions of the Bloch states are $\boldsymbol{k}$ dependent in the ground state of a narrow, roughly half-filled superconducting band (this striking feature of the Bloch electrons suggests interpreting superconductivity as " $\boldsymbol{k}$ space magnetism" [12]). The Bloch functions $\varphi_{\boldsymbol{k}, q, \boldsymbol{m}}(\boldsymbol{r}, t)$ are labeled, as usual, by the wave vector $\boldsymbol{k}$ and the band index $q$, but no longer by the electron spin $\boldsymbol{s}$ since the spin direction is $\boldsymbol{k}$ dependent. They are rather labeled by the "crystal spin" $\boldsymbol{m}$ defined within the NHM [2] [3].

In a system with $\boldsymbol{k}$ dependent spin directions the electrons couple to crystalspin-1 boson excitations in order that the total crystal-spin angular-momentum is conserved during the ever-present scattering processes in the electron system, see Section 3.2 of Ref. [4]. At low temperatures, the electrons try to occupy a state in which the electrons alone satisfy the conservation of spin-angular momentum. The only fixed spin directions in a superconducting band are those of a Bloch state $\varphi_{\boldsymbol{k}, q, \boldsymbol{m}}(\boldsymbol{r}, t)$ and its time-inverted state,

$$
\varphi_{-\boldsymbol{k}, q,-\boldsymbol{m}}(\boldsymbol{r}, t)=K \varphi_{\boldsymbol{k}, q, \boldsymbol{m}}(\boldsymbol{r}, t),
$$

since both states have exactly opposite spin directions. $K$ denotes the operator of time inversion.

At low temperatures, the electrons form Cooper pairs consisting in each case of a Bloch state and its time inverted state. When all the electrons of the superconducting band form Cooper pairs with zero total spin-angular momentum, the conservation of spin angular-momentum is satisfied in the electron system alone, see the group-theoretical substantiation in Section 3.2 of Ref. [4].

The mechanism of Cooper pair formation can be described in terms of constraining forces produced by the crystal-spin-1 boson excitations, see Section 3.3 of Ref. [4]. As illustrated in Figure 3 of Ref. [13], these constraining forces behave like classical constraining forces produced by springs: Let $\mathcal{P}$ be the Hilbert space spanned by the electron states in the superconducting band and $\mathcal{P}^{0}$ the subspace of $\mathcal{P}$ in which all the electrons form Cooper pairs. Assume all the electrons of the superconducting band initially to be in $\mathcal{P}^{0}$. Whenever 
two electrons are scattered out of $\mathcal{P}^{0}$, a crystal-spin-1 boson pair is excited which can only be reabsorbed when the electrons are scattered in such a way that again they lie in $\mathcal{P}^{0}$. Hence, the crystal-spin-1 bosons behave like "springs" that push the electrons back into $\mathcal{P}^{0}$. So we may speak of "spring-mounted" Cooper pairs.

\subsection{Superconducting Band in an Outer Magnetic Field}

Now assume an outer magnetic field to be switched on. An absolutely consistent mathematical description of superconductivity in an outer magnetic field would require to show that the spin-dependent Wannier functions in a superconducting band may be chosen symmetry-adapted even in the presence of an outer magnetic field, as it has been carefully established [2] for the field-free case. Though the symmetry of the Bloch and Wannier functions is, in principle, known in magnetic fields [14] [15] [16], this would be a complicated and, as I believe, physically needless task. Instead, we should keep in mind that the spin-dependent Wannier functions represent localized electron states that really exist in the material. These localized states clearly are adapted the symmetry of the electron system. For this reason we can assume that the spin-dependent Wannier functions in a superconducting band may be chosen adapted to the symmetry of the electron system even in the presence of an outer magnetic field. In this context, the symmetry of the electron system comprises the inner timeinversion symmetry as shall be defined in Section 4.2.

\section{The Hamiltonian in an Uniform Magnetic Field}

The Hamiltonian of an electron in a solid state and in a uniform external magnetic field has the form

$$
\mathcal{H}=\frac{1}{2 m}\left(\boldsymbol{p}+\frac{e}{c} \boldsymbol{A}\right)^{2}+V(\boldsymbol{r}),
$$

where

$$
\boldsymbol{p}=\boldsymbol{p}_{\text {kin }}-\frac{e}{c} \boldsymbol{A}
$$

is the operator of the generalized momentum, $m$ is the electron mass, $e$ the proton charge, $V(\boldsymbol{r})$ is the periodic potential, $\boldsymbol{p}_{\text {kin }}$ is the so-called "kinetic momentum", and A denotes the operator of the vector potential [17] [18]. An additional term standing for the energy of the electron spins in the magnetic field is neglected.

The translation operators in the magnetic field may be written as

$$
T(\boldsymbol{R})=\mathrm{e}^{-i \boldsymbol{R} \cdot \boldsymbol{p} / \hbar},
$$

where $\boldsymbol{R}$ is a lattice vector and $\boldsymbol{p}$ is the generalized momentum given in Equation (3) [14]. Since the translation operators $T(\boldsymbol{R})$ commute with $\mathcal{H}$ [14],

$$
[T(\boldsymbol{R}), \mathcal{H}]=0
$$


we may label the eigenfunctions of $\mathcal{H}$ by the generalized impulse $\boldsymbol{p}$ and write

$$
\mathcal{H} \varphi_{p, q, \boldsymbol{m}}(\boldsymbol{r}, t)=E_{\boldsymbol{p}, q, \boldsymbol{m}} \varphi_{\boldsymbol{p}, q, \boldsymbol{m}}(\boldsymbol{r}, t),
$$

as it was already performed by Onsager to interpret the de Haas-van Alphen Effect [19]. $q$ still is the band index and $t$ is the spin coordinate. Just as in the field-free case, $\boldsymbol{m}$ does not stand for the electron spin but denotes the crystal spin since the spin direction depends on $\boldsymbol{p}$ in a narrow, roughly half-filled superconducting band.

\section{Cooper Pairs within an Outer Magnetic Field}

\subsection{The Inner Time-Inversion}

Consider a superconducting sample within an external magnetic field generated by Helmholtz coils fare away from the sample. As is well-known, the electron system within the sample is invariant under time inversion only if additionally the magnetic field $\boldsymbol{B}$ and, hence, the vector potential $\boldsymbol{A}$ is inverted,

$$
K^{-1} A K=-A \text {, }
$$

where $K$ denotes the operator of time inversion, see, e.g., Ref. [18]. This important phenomenon can be understood already in classical physics: in a magnetic field, the Lorentz force generates within the sample a circular motion of the electrons. An inversion of the time of the system produces a circular motion of the opposite direction of rotation. In a fixed magnetic field, however, the Lorentz force generates in any case circular motions of the same sense of rotation. Hence, a time inverted circular motion of the electrons may exist only in the inverted magnetic field. Thus, an inversion of the time requires that the experimentalist additionally reverses the polarity of the battery connected with the Helmholtz coils. Hence, $K$ is not a symmetry operation of the electron system.

This problem has been overcome for special sheared solids [20] and for reversible microscopic systems [21]. In the present paper, however, we do not consider the standard time-inversion represented by $K$ connected with the complete system consisting of both the superconducting sample and the Helmholtz coils. Instead, we see the superconducting sample as an inner isolated system within a fixed magnetic potential $\boldsymbol{A}$ produced by the outer Helmholtz coils. We define an operator $\bar{K}$ inverting the time $\tau$ within the inner electron system,

$$
\tau \rightarrow-\tau,
$$

without changing the outer magnetic field,

$$
\bar{K}^{-1} A \bar{K}=A .
$$

Thus, this operator $\bar{K}$ of the "inner time inversion" has the same effect as $K$ [22] on the kinetic momenta $\boldsymbol{p}_{\text {kin }}$, the spins $\boldsymbol{s}$ and the positions $\boldsymbol{r}$ of the inner electrons,

$$
\bar{K}^{-1} \boldsymbol{p}_{k i n} \bar{K}=-\boldsymbol{p}_{\text {kin }},
$$




$$
\begin{gathered}
\bar{K}^{-1} \boldsymbol{s} \bar{K}=-\boldsymbol{s}, \\
\bar{K}^{-1} \boldsymbol{r} \bar{K}=\boldsymbol{r} .
\end{gathered}
$$

In contrast to the standard time inversion $K$, however, it does not invert the sense of rotation of the circular motions produced by the outer Lorentz force. Also $\bar{K}$ is an anti-linear operator because it complies with the conditions given in Section 26 in the textbook of E. P. Wigner [22].

\subsection{The Total Momentum of a Cooper Pair}

With Equation (3) the Hamiltonian may be written as

$$
\mathcal{H}=\frac{1}{2 m}\left(\boldsymbol{p}_{\text {kin }}\right)^{2}+V(\boldsymbol{r})
$$

showing immediately that $\bar{K}$ commutes with $\mathcal{H}$,

$$
\bar{K}^{-1} \mathcal{H} \bar{K}=\mathcal{H},
$$

if we continue to neglect the energy of the electron spins in the magnetic field. From this result follows the significant insight that the inner time-inversion $\bar{K}$ is a symmetry operation of the inner electron system.

As argued in Section 2.2, the magnetic Wannier functions are adapted to the inner time-inversion just as they are adapted to the standard time inversion in the field-free case. As a consequence, the operator $\bar{K}$ acts on the crystal spin $\boldsymbol{m}$ in the same way as it acts on the spin $s$,

$$
\bar{K}^{-1} \boldsymbol{m} \bar{K}=-\boldsymbol{m},
$$

as it has been shown for the zero-field case in Section 7.3.1 of Ref. [2].

Since the operator $\bar{K}$ commutes with $\mathcal{H}, \bar{K}$ transforms an eigenstate $\varphi_{\boldsymbol{p}, q, \boldsymbol{m}}(\boldsymbol{r}, t)$ of $\mathcal{H}$ into a new eigenstate of $\mathcal{H}$,

$$
\varphi_{\boldsymbol{p}^{\prime}, q,-\boldsymbol{m}}(\boldsymbol{r}, t)=\bar{K} \varphi_{\boldsymbol{p}, q, \boldsymbol{m}}(\boldsymbol{r}, t),
$$

associated with the same energy, where

$$
\boldsymbol{p}^{\prime}=\bar{K}^{-1} \boldsymbol{p} \bar{K} .
$$

With Equations (3), (9) and (10) we obtain

$$
\boldsymbol{p}^{\prime}=-\boldsymbol{p}_{\text {kin }}-\frac{e}{c} \boldsymbol{A} .
$$

Remember that the direction of the electron spins depends on $p$ in a narrow, roughly half-filled superconducting band. Just as in the field-free case, the constraining forces produced by the crystal-spin-1 excitations generate Cooper pairs with exactly vanishing total spin-angular momentum. Equation (11) ensures that the spins of the two electrons occupying the states $\varphi_{p^{\prime}, q,-m}(r, t)$ and $\varphi_{p, q, m}(r, t)$ in Equation (16) are exactly anti-parallel. Consequently, these two states (and only these two states) can form Cooper pairs. (The basic Equation (125) of Ref. [2] ensuring a vanishing total spin-angular momentum is satisfied even in an outer magnetic field if we replace $\boldsymbol{k}$ by $\boldsymbol{p},-\boldsymbol{k}$ by $\boldsymbol{p}^{\prime}$, and $K$ by $\bar{K}$ in the derivation of this equation.) 
Hence, in a magnetic field, the total momentum $p_{c}$ of the two electrons forming a Cooper pair in a superconducting band does not vanish, but has the value

$$
\boldsymbol{p}_{c}=\boldsymbol{p}+\boldsymbol{p}^{\prime}=-\frac{2 e}{c} \boldsymbol{A} .
$$

This equation gives the exact total momentum of a Cooper pair within an outer magnetic field. It shall be interpreted in the following Section 5.

\section{The London Equations}

Equation (19) shows that the kinetic momenta of the two Bloch states forming a Cooper pair cancel each other. However, the term $-\frac{2 e}{c} A$ indicates that the Lorentz force still is active and forces the two electrons to perform a circular motion with the same sense of rotation each. Because the two electrons move on different orbitals, the probability to meet an electron at a certain position $\boldsymbol{r}$ is different for the two electrons and, hence, their average total kinetic momentum $\left\langle\boldsymbol{p}_{c, k i n}\right\rangle$ at $\boldsymbol{r}$ needs not vanish. Thus, the electron pair with the momentum $\boldsymbol{p}_{c}$ may produce a $\boldsymbol{r}$ dependent electrical current $\boldsymbol{j}_{c}$ which is defined by the symmetry of the system.

To determine $\boldsymbol{j}_{c}$, we rewrite Equation (19) as

$$
\boldsymbol{p}_{c}=-\frac{e}{c} \boldsymbol{A}-\frac{e}{c} \boldsymbol{A}
$$

showing that $\boldsymbol{p}_{c}$ has the form given in Equation (3) if we interpret one of the addends as the average kinetic momentum

$$
\left\langle\boldsymbol{p}_{c, k i n}\right\rangle=-\frac{e}{c} \boldsymbol{A}
$$

of an one-electron state.

Due to this interpretation (21), the operator

$$
T(\boldsymbol{R})=\mathrm{e}^{-i \boldsymbol{R} \cdot \boldsymbol{p}_{c} / \hbar}
$$

becomes a translation operator commuting with $\mathcal{H}$, and, hence, the oneelectron state with the momentum $\boldsymbol{p}_{c}$ becomes an eigenstates of $\mathcal{H}$. Consequently, an electrical current represented by this state has physical reality.

Thus, the contribution of one Cooper pair to the electric current amounts to

$$
\boldsymbol{j}_{c}=\frac{e}{m}\left\langle\boldsymbol{p}_{c, k i n}\right\rangle=-\frac{e^{2}}{m c} \boldsymbol{A} .
$$

$\boldsymbol{j}_{c}$ is invariant under the inner time-inversion $\bar{K}$ because it is originally defined by Equation (19), i.e., by the outer vector potential $\boldsymbol{A}$.

Equation (23) is the result of this paper. It contains both London equations [10] in a compact form, see Equation (1.8) in the textbook of M. Tinkham [11].

\section{Conclusions}

This paper provides evidence that the constraining forces causing the formation of Cooper pairs in narrow, roughly half-filled superconducting bands are also 
responsible for the Meissner effect. In the framework of the nonadiabatic Heisenberg model, the Meissner effect is an intrinsic part of superconductivity.

Hirsch [9] argues that neither BCS theory nor London electrodynamic theory describes superconductivity. But, he adds that parts of both BCS theory and London theory are undoubtedly correct. From my point of view, I can confirm this strong statement of Hirsch. However, I specify that BCS theory as well as London theory are correct if the constraining forces operating in narrow, roughly half-filled superconducting bands are present.

\section{Acknowledgements}

I am very indebted to Guido Schmitz for his support of my work.

\section{References}

[1] Krüger, E. (2001) Physical Review B, 63, 144403-1-13. https://doi.org/10.1103/PhysRevB.63.144403

[2] Krüger, E. and Strunk, H.P. (2015) Symmetry, 7, 561-598. https://doi.org/10.3390/sym7020561

[3] Krüger, E. (2001) Journal of Superconductivity, 14, 469-489. (Please note that in this paper the term "superconducting band" was abbreviated by " $\sigma$ band".) https://doi.org/10.1023/A:1012231428443

[4] Krüger, E. (2010) Journal of Superconductivity and Novel Magnetism, 23, 213-223. https://doi.org/10.1007/s10948-009-0518-1

[5] Krüger, E. (1989) Physica Status Solidi B, 156, 345-354. https://doi.org/10.1002/pssb.2221560135

[6] Krüger, E. (1984) Physical Review B, 30, 2621-2633. https://doi.org/10.1103/PhysRevB.30.2621

[7] Bardeen, J., Cooper, L.N. and Schrieffer, J.R. (1957) Physical Review, 108, 1175. https://doi.org/10.1103/PhysRev.108.1175

[8] Meissner, W. and Ochsenfeld, R. (1933) Naturwissenschaften, 21, 787-788. https://doi.org/10.1007/BF01504252

[9] Hirsch, J.E. (2012) Physica Scripta, 85, 035704. https://doi.org/10.1088/0031-8949/85/03/035704

[10] London, F. and London, H. (1935) Physica, 2, 341-354.

[11] Tinkham, M. (1996) Introduction to Superconductivity. 2nd Edition, McGraw-Hill, New York

[12] Krüger, E. (2002) Superconductivity Interpreted as k-Space Magnetism. arXiv:cond-mat/0209069

[13] Krüger, E. (2002) Journal of Superconductivity, 15, 105-108. https://doi.org/10.1023/A:1014663201068

[14] Brown, E. (1964) Physical Review, 133, A1038-A1044. https://doi.org/10.1103/PhysRev.133.A1038

[15] Wannier, G.H. (1962) Reviews of Modern Physics, 34, 645-655. https://doi.org/10.1103/RevModPhys.34.645

[16] Fischbeck, H.J. (1970) Physica Status Solidi (B), 38, 11-62.

[17] Kittel, C. (1986) Introduction to Solid State Physics. 6th Edition, Wiley, New York. 
[18] Landau, L.D. and Lifšic, E.M. (2007) Quantenmechanik. Deutsch, Frankfurt am Main.

[19] Onsager, L. (1952) The London, Edinburgh, and Dublin Philosophical Magazine and Journal of Science, 43, 1006-1008. https://doi.org/10.1080/14786440908521019

[20] Bonella, S., Ciccotti, G. and Rondoni, L. (2014) EPL, 108, Article ID: 60004. https://doi.org/10.1209/0295-5075/108/60004

[21] Dal Cengio, S. and Rondoni, L. (2016) Symmetry, 8, 73. https://doi.org/10.3390/sym8080073

[22] Wigner, E.P. (1964) Group Theory and Its Application to the Quantum Mechanics of Atomic Spectra. Academic Press, New York.

Submit or recommend next manuscript to SCIRP and we will provide best service for you:

Accepting pre-submission inquiries through Email, Facebook, LinkedIn, Twitter, etc. A wide selection of journals (inclusive of 9 subjects, more than 200 journals)

Providing 24-hour high-quality service

User-friendly online submission system

Fair and swift peer-review system

Efficient typesetting and proofreading procedure

Display of the result of downloads and visits, as well as the number of cited articles

Maximum dissemination of your research work

Submit your manuscript at: http://papersubmission.scirp.org/

Or contact jmp@scirp.org 